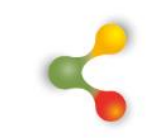

REVUE HYBRIDE DE L’ÉDUCATION

\title{
Pratiques enseignantes \\ Enseigner les soins infirmiers en temps de pandémie
}

Auteures

Camille Godue-Couture, candidate à la maitrise en éducation, Université du Québec à Chicoutimi, Canada,

camille.godue-couture1@uqac.ca

Guylaine Harvey, enseignante en soins infirmiers, cégep de Saint-Félicien, Canada,

gharvey@cegepstfe.ca 


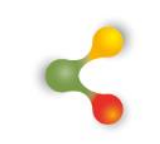

\section{REVUE HYBRIDE DE L'ÉDUCATION}

\section{Résumé}

La pandémie de la COVID-19 a forcé l'adaptation rapide des pratiques enseignantes et plusieurs changements ont été apportés durant l'année 2020 afin de s'assurer qu'un enseignement de qualité soit fourni à tous les étudiants, tout en respectant les restrictions mises en vigueur par le gouvernement du Québec. Cet article présente conséquemment les adaptations et modifications effectuées en raison de la pandémie par Guylaine Harvey et ses collègues du département de soins infirmiers au cégep de Saint-Félicien durant les sessions d'hiver et d'automne 2020.

Mots-clés : soins infirmiers; pandémie; collégial; adaptation; enseignement à distance 


\section{$\&$}

\section{REVUE HYBRIDE DE L'ÉDUCATION}

\section{Mise en contexte}

Les pratiques enseignantes ont toujours été en constante évolution. Elles ont parfois été dictées par l'époque, à d'autres moments, elles ont évolué, influencées par l'État et les réformes de l'éducation, ou encore, elles sont l'initiative d'un enseignant. En 2020, une situation hors du commun a bouleversé plusieurs secteurs, dont celui de l'éducation. La pandémie de la COVID-19 a forcé le gouvernement du Québec à imposer un confinement quasi total à sa population en mars 2020. De ce fait, tous les établissements scolaires ont dû revoir leurs pratiques afin de les adapter à cette nouvelle réalité pour fournir une éducation adéquate à tous les apprenants.

Conséquemment, le cégep de Saint-Félicien, tout comme les autres collèges du Québec, a été invité à apporter d'importants changements pour permettre aux étudiants de terminer leurs sessions à distance. Les enseignants n'ont eu que très peu de temps pour adapter leurs plans de cours et ajuster leurs méthodes d'enseignement.

À la fin de cette session particulière, les enseignants ont commencé à planifier la prochaine rentrée scolaire. Les mesures sanitaires étant incertaines, tous les scénarios étaient envisagés. Finalement, à la suite des consignes imposées par le gouvernement, la session d'automne 2020 au cégep de Saint-Félicien a pu avoir lieu en présentiel, les cours théoriques comme pratiques étaient de nouveau possibles. Les stages en milieu de pratique ont également pu reprendre. En octobre, voyant le nombre de cas augmenter, le gouvernement du Québec a toutefois attribué au SaguenayLac-Saint-Jean le palier d'alerte maximale. Ce changement a eu pour effet de modifier les consignes sanitaires et, par le fait même, les mesures mises en place par les institutions scolaires de la région.

Sans l'ombre d'un doute, la pandémie a donné lieu à des situations hors du commun qui ont forcé l'innovation des pratiques enseignantes. Cet article présente les adaptations et initiatives que madame Harvey et ses collègues du département des soins infirmiers au cégep de Saint-Félicien ont mises en place afin de permettre aux étudiants de voir tous les contenus notionnels essentiels dans ces conditions exceptionnelles.

Madame Guylaine Harvey est infirmière depuis 1992. Elle a travaillé durant 16 ans comme infirmière dans différents départements et milieux. En 2000, elle a décidé de commencer son baccalauréat en soins infirmiers afin de bonifier sa formation. Quelques années plus tard, l'occasion de travailler au cégep de Saint-Félicien s'est présentée. Elle a d'abord travaillé au laboratoire afin de superviser les pratiques des étudiants.

Par la suite, un poste d'enseignant en médecine-chirurgie lui a été proposé, domaine dans lequel elle était déjà formée. Elle enseigne depuis plus d'une dizaine d'années le cours Médecine-Chirurgie pour les étudiants 


\section{$\&$}

\section{REVUE HYBRIDE DE L'ÉDUCATION}

de deuxième année en soins infirmiers au cégep de Saint-Félicien. Madame Harvey a à cœur la réussite de ses étudiants. Elle veut transmettre l'amour et la passion qu'elle porte pour sa profession. Pour elle, il est important d'offrir aux étudiants une formation qui répond aux normes et aux besoins de la profession infirmière, mais qui est également adaptée à la réalité des étudiants.

Au cégep de Saint-Félicien, le département de soins infirmiers comporte neuf enseignants. Cette petite équipe a su se soutenir et s'entraider depuis le début de la pandémie de la COVID-19. Une entrevue a été réalisée avec madame Harvey afin qu'elle partage les adaptations mises en place par son équipe pour favoriser l'apprentissage des étudiants au fil des différentes sessions marquées par la pandémie.

\section{Déroulement}

La pandémie a forcé les établissements scolaires à modifier leurs pratiques très rapidement. Au cégep de Saint-Félicien, comme ailleurs, les enseignants ont dû adapter leurs cours afin de respecter les mesures sanitaires. Enseigner les soins infirmiers à distance n'est pas facile à envisager puisque cette profession requiert un contact direct avec la clientèle. Un grand nombre de cours comporte également un volet pratique puisque plusieurs manipulations techniques sont nécessaires à maitriser pour pouvoir exercer la profession. En outre, le programme comporte plusieurs stages. Durant ceux-ci, les étudiants accompagnés par leurs enseignants se rendent en milieux hospitaliers pour mettre en application les apprentissages acquis et peaufiner leurs formations. L'équipe d'enseignants du département des soins infirmiers a su mettre en œuvre sa créativité afin d'adapter leurs enseignements selon les limites imposées par la pandémie.

\section{Hiver 2020}

La session d'hiver 2020 avait commencé sans embuches et se déroulait comme prévu. C'est environ à la mi-session que tout a changé. Des mesures drastiques ont été mises en place pour le gouvernement du Québec afin de limiter la propagation du virus. Les cours ont alors été suspendus durant plus de deux semaines. Les enseignants en soins infirmiers du cégep de Saint-Félicien ont alors profité de ces deux semaines pour préparer la transition en mode virtuel. Cela leur a permis de modifier leurs plans de cours et d'envisager la façon de pouvoir transmettre tous les contenus notionnels des cours à distance.

Durant ces deux semaines de transition, les conseillers pédagogiques avaient fait parvenir aux enseignants des formations en ligne, leur permettant de mieux comprendre les outils numériques mis à leur disposition afin qu'ils soient en mesure d'effectuer leurs cours à distance. 


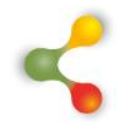

\section{REVUE HYBRIDE DE L'ÉDUCATION}

Passer à un mode d'enseignement virtuel n'a pas été facile pour tous. Alors que certains enseignants avaient une plus grande aisance et utilisaient déjà la plateforme Moodle pour distribuer leurs documents de cours, d'autres ont dû apprendre le tout de $A$ à $Z$ en l'espace de deux semaines.

Moodle est une plateforme d'apprentissage en ligne sur laquelle on peut, entre autres, déposer des documents et des hyperliens, créer des discussions de groupe et faire des sondages (Rice et William, 2006).

Durant la session d'hiver 2020, tous les cours théoriques ont été enseignés via la plateforme Zoom. Celle-ci a permis d'effectuer les cours en visioconférence. Les examens ont aussi dû être réalisés à distance. Ils étaient distribués sur Moodle et les enseignants pouvaient superviser les étudiants en direct par vidéoconférence.

Le plus grand enjeu vécu par le département des soins infirmiers du cégep de Saint-Félicien a été l'impossibilité de se rendre dans les milieux de stage. La technique de soins infirmiers est un programme qui se réalise en trois ans. Les étudiants des différentes années avaient déjà commencé leurs stages respectifs, mais aucun n'était complété. Lorsque l'enseignement est passé à distance, les stages ont dû prendre fin. Pour pallier les heures manquantes, madame Harvey et ses collègues ont décidé de se tourner vers deux types de formations en ligne interactives : la formation Priim@ (https://www.formationpriima.ca/) et celles offertes par l'Ordre des infirmières et infirmiers du Québec (OIIQ).

La formation Priim@ est un programme qui permet la consolidation des apprentissages par l'entremise d'une plateforme en ligne afin de mieux préparer les étudiants à la passation de l'examen professionnel de l'OIIQ (Formation Priim@, s.d). Cette formation a été développée par les cégeps de Chicoutimi et de Jonquière, ainsi que l'Université du Québec à Chicoutimi. Cette formation requiert des licences pour y participer. La direction du cégep de Saint-Félicien a alors débloqué un budget afin que les étudiants en soins infirmiers puissent y accéder. Cette formation est interactive et permet aux étudiants de tester leurs compétences de façon théorique et pratique par le biais de jeux vidéo sérieux, d'aide-mémoire, de jeux-questionnaires, de calculs, etc. (Formation Priim@, s.d) Ce qui était intéressant pour les enseignants est qu'ils étaient en mesure de suivre, à distance, la progression de leurs étudiants tout au long de leur formation Priim@. Bien entendu, les étudiants n'avaient pas à réaliser la totalité de la formation. Chaque enseignant avait visionné le contenu de la formation selon ses contenus de cours et le cheminement de ses étudiants. Ils étaient donc en mesure de guider leurs étudiants au travers des différents contenus proposés, à la lumière des notions vues en classe.

Les enseignants du département de soins infirmiers se sont également tournés vers les formations en ligne offertes par l'OIIQ. Ils ont 


\section{$\&$}

\section{REVUE HYBRIDE DE L'ÉDUCATION}

procédé de la même manière que pour la formation Priim@, c'est-à-dire qu'ils ont choisi le contenu de la formation selon leurs cours et l'année d'études de leurs étudiants.

Dans le but de bonifier l'apprentissage des étudiants, des vidéos issus des éditeurs des manuels de référence utilisés en classe étaient aussi disponibles.

Madame Harvey a voulu enrichir davantage l'apprentissage de ses étudiants. Pour compenser l'absence de stages, en plus des formations en ligne, elle a décidé de concevoir des mises en situations uniques pour chaque étudiant. Elle a envoyé, à chacun d'entre eux, une mise en situation réaliste d'un patient à l'hôpital. L'étudiant avait accès aux informations du patient, à ses résultats de tests, etc. Madame Harvey planifiait ensuite une vidéoconférence d'une heure avec chaque étudiant. Durant celle-ci, elle posait des questions aux étudiants et avançait étape par étape comme s'ils étaient réellement sur le département de chirurgie à l'hôpital. Au fur et à mesure, madame Harvey transmettait les données du patient à l'étudiant et celui-ci devait réagir en fonction de l'information qu'il recevait. Cette expérience a été très appréciée par les étudiants, car elle leur a permis de se visualiser sur le terrain et de mettre en application leurs apprentissages de manière plus concrète.

Les examens de fin de session ont également eu lieu à distance. Les étudiants recevaient les examens sur Moodle et se connectaient en vidéoconférence sur Zoom. Les enseignants étaient alors en mesure de les superviser et de répondre à leurs questions. II a été difficile de superviser adéquatement les étudiants lors des examens à distance. En effet, il était difficile pour eux de percevoir si des étudiants trichaient, même si leurs caméras devaient rester ouvertes. Ils sont incertains s'il y a eu de la tricherie ou non durant les examens de la session d'hiver 2020, mais souhaitent ne plus recommencer l'expérience. Ils vont prioriser, si possible, les examens en présentiel.

Bref, ces modifications aux plans de cours des enseignants ont permis aux étudiants d'apprendre tous les contenus notionnels et de développer les compétences requises pour chaque cours. Les changements effectués durant la session d'hiver 2020 n'auraient pu être possibles sans la collaboration de la direction et de tous les enseignants en soins infirmiers du cégep de Saint-Félicien.

\section{Automne 2020}

Madame Harvey et ses collègues ont commencé à préparer la rentrée scolaire en juin. Les mesures sanitaires étaient encore incertaines pour la session d'automne 2020 , tous se préparaient donc à toute éventualité. Finalement, le gouvernement du Québec a permis aux 


\section{$\&$}

\section{REVUE HYBRIDE DE L'ÉDUCATION}

institutions scolaires d'enseigner en présentiel, tout en respectant les consignes sanitaires et les mesures de distanciation sociale.

Comme mentionné précédemment, la technique de soins infirmiers comporte un grand nombre de séances pratiques durant lesquelles les étudiants apprennent des gestes techniques importants (ex. : comment faire une prise de sang, comment installer une sonde urinaire ou comment effectuer des ponctions veineuses). Voulant se préparer à toute éventualité, les enseignants du département de soins infirmiers du cégep de Saint-Félicien se trouvaient face à un problème. Comment peut-on enseigner diverses méthodes de soins, qui nécessitent de la pratique, en formation à distance? L'équipe d'enseignants en soins infirmiers a alors entrepris plusieurs adaptations à leur enseignement, lesquelles seront présentées dans les lignes qui suivent.

Ils ont d'abord décidé de créer des vidéos pour chaque méthode à enseigner. Une personne était désignée pour filmer, une autre s'occupait de la description et une dernière personne réalisait la technique appropriée selon la méthode de soins expliquée. Les vidéos ont ensuite été déposées sur Moodle et ont été rendues accessibles à tous les étudiants du département des soins infirmiers. Alors que les vidéos permettaient aux étudiants de première année de s'initier à ces méthodes, elles étaient utiles pour ceux en dernière année qui désiraient les réviser ou bonifier leurs pratiques.

Les enseignants en soins infirmiers ont mis énormément de temps à la préparation et à la création de ces vidéos pédagogiques. Même si les laboratoires, les cours pratiques et ceux théoriques ont finalement pu être enseignés en présentiel, ils ont tout de même utilisé leurs vidéos. Leurs efforts ont porté fruit, puisque les vidéos réalisées par les enseignants se sont avérées appréciées par les étudiants. Les enseignants désirent continuer à en créer pour pouvoir les utiliser lors des sessions ultérieures.

En technique de soins infirmiers, les étudiants ont généralement accès à des laboratoires dans lesquels ils peuvent venir pratiquer les techniques de soin apprises en classe.

Afin de bien se préparer pour la rentrée, les enseignants du département de soins infirmiers du cégep de Saint-Félicien avaient mis sur pied un système de boites individuelles. Ces boites ont été identifiées au nom des étudiants et chacune contenait le matériel nécessaire pour que l'étudiant soit en mesure de pratiquer les techniques apprises en classe, même à la maison. Même si les laboratoires ont finalement pu avoir lieu, le système de boites individuelles s'est avéré fort pratique. Ce système a permis une meilleure gestion de l'équipement dans le laboratoire et un bon respect des mesures sanitaires au sein du département. Lorsque les étudiants arrivent au laboratoire, ils prennent le bac identifié à leur nom et travaillent avec leur matériel. 


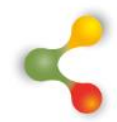

\section{REVUE HYBRIDE DE L'ÉDUCATION}

Le système de bacs a également été utile lors de cas de COVID-19 auprès des étudiants en soins infirmiers. Étant donné que les boites contenaient tout le matériel nécessaire à la pratique, si un étudiant était dans l'obligation de s'isoler, son bac pouvait être apporté, par un autre étudiant, à son lieu de résidence. L'étudiant mis en isolement était alors en mesure de pratiquer à partir de chez lui.

À l'automne 2020, les stages ont également dû être adaptés. Avant la pandémie, le centre intégré universitaire de santé et de services sociaux du Saguenay-Lac-Saint-Jean (CIUSSS) permettait aux enseignants d'avoir un maximum de sept étudiants avec eux sur le département dans lequel le stage était effectué. Depuis la session d'automne 2020, le nombre maximal d'étudiants permis par enseignant pour les stages est désormais de quatre. Cette mesure a pour but de limiter le nombre de personnes présentes sur chaque département afin d'éviter la propagation du virus. Le CIUSSS a également demandé au cégep de Saint-Félicien d'augmenter le nombre de plages horaires utilisées pour les stages.

Par conséquent, les enseignants du département de soins infirmiers doivent donc passer deux fois plus de temps à superviser et enseigner les stages. Leurs horaires ont donc changé et les enseignants doivent maintenant travailler de soir et durant certaines fins de semaine pour pouvoir être en mesure d'offrir le même nombre d'heures de stage à tous les étudiants.

De plus, le CIUSSS a demandé au département des soins infirmiers du cégep de Saint-Félicien d'innover et d'essayer d'être moins présents sur les départements dans les différents milieux hospitaliers. Madame Harvey et ses collègues ont conséquemment décidé de faire vivre à leurs étudiants une simulation d'une journée type dans le département où leur stage avait lieu. Cette journée remplaçait une des journées de stages prévues à l'horaire.

Cette journée avait lieu dans les laboratoires du cégep de SaintFélicien. Les enseignants se sont débrouillés pour que tout soit le plus réel possible : les dossiers des patients, les formulaires, les examens, etc. Si le stage avait lieu le soir, alors les étudiants devaient changer l'heure de leurs montres. Avec l'aide de l'enseignant, les étudiants ont été en mesure de vivre la routine du département où leur stage avait lieu, mais au cégep. Ces simulations ont donc servi à titre de première journée dans leur milieu de stage. Cette journée a été grandement appréciée des étudiants. Cela leur a permis d'être plus confiants à leur arrivée dans leur milieu de stage. Effectivement, cette façon de faire réduit le nombre d'heures passées en milieu clinique, mais favorise l'intégration des étudiants et réduit le stress associé à la première journée de stage.

En somme, beaucoup d'adaptations ont été nécessaires de la part des enseignants de la technique en soins infirmiers du cégep de Saint- 


\section{$\&$}

\section{REVUE HYBRIDE DE L'ÉDUCATION}

Félicien durant la pandémie. Ces modifications ont eu des effets sur les enseignants et leurs pratiques, mais également sur les étudiants.

\section{Apports et prospectives}

Les pratiques enseignantes ayant été rapidement bouleversées par la pandémie, plusieurs acteurs ont apporté leur contribution et leur support afin d'aider les enseignants à adapter leur enseignement à cette nouvelle réalité. Pour madame Harvey, le soutien de ses collègues demeure un atout non négligeable. Elle souligne également l'importance de la communication et de l'entraide entre ses collègues du département. Cette bonne coopération a contribué à faire diminuer le stress créé par la pandémie, en plus de rapprocher les enseignants du département de soins infirmiers.

La direction a aussi été un acteur important à la réalisation des changements apportés durant l'année 2020. Elle a permis la modification rapide des plans de cours et a offert un soutien financier pour que les formations en ligne interactives soient accessibles aux étudiants et aux enseignants pour combler les heures prévues de stages. La direction a également soutenu le projet de boites individuelles et a soutenu l'achat des bacs et du matériel nécessaire à chaque étudiant. La direction a été très accommodante et ouverte aux changements. Elle a également su faire confiance aux initiatives des enseignants dans le but de bonifier l'expérience et les apprentissages des étudiants. Sans le soutien de la direction, les adaptations apportées par les enseignants en soins infirmiers auraient été difficiles à réaliser.

Le département des soins infirmiers mentionne également l'apport des conseillers pédagogiques et des techniciens en communication et informatique. Ils ont été d'une grande aide durant la période de transition en mars 2020 et ont mis sur pied des formations essentielles afin que les enseignants se sentent à l'aise d'enseigner à distance. L'université TÉLUQ, université publique qui offre seulement de l'enseignement à distance, a partagé certaines formations sur l'enseignement à distance qui ont été à leur tour partagées par les conseillers pédagogiques du cégep de SaintFélicien.

Le CIUSSS a également contribué au succès des adaptations nécessaires en temps de pandémie. Madame Harvey souligne avoir apprécié l'accompagnement et la communication entre le département de soins infirmiers et le CIUSSS. Le CIUSSS a, par moments, donné de judicieux conseils au département et a formé les enseignants en ce qui a trait aux nouveaux protocoles sanitaires dans les milieux hospitaliers dus à la pandémie. Plusieurs étudiants en soins infirmiers travaillent également dans les centres d'hébergement de soins de longue durée (CHSLD) de la région. Le CIUSS étant leur employeur, il est demeuré flexible et a accordé des congés lorsque les étudiants avaient des stages la fin de semaine. Ils 


\section{8}

\section{REVUE HYBRIDE DE L'ÉDUCATION}

ont aussi accordé des congés aux étudiants travaillant en CHSLD avant et après leurs stages afin de limiter la propagation du virus d'un établissement à l'autre. Pour madame Harvey, il y a eu une belle complicité et un réel engagement autant du CIUSSS que du département des soins infirmiers du cégep de Saint-Félicien afin que tout se déroule le mieux possible.

Finalement, les étudiants ont dû se montrer très conciliants durant ces deux sessions afin de s'ajuster à l'enseignement à distance et aux modifications proposées dans leurs sessions d'études. Selon madame Harvey, les étudiants se sont montrés très résilients et motivés. Il y a certes un léger retard qui a été observé au début de la session d'automne 2020, principalement en ce qui concerne l'autonomie et la rapidité des mouvements techniques des étudiants. Par contre, elle considère que les enseignants doivent être compréhensifs et conciliants puisque les étudiants ont tout de même manqué une bonne portion de laboratoires pratiques durant la session d'hiver 2020. Elle considère que le retard sera réduit, voire inexistant lors de la prochaine session.

\section{Des adaptations qui vont perdurer}

La pandémie a forcé l'innovation des pratiques enseignantes. Durant cette grande période d'adaptation, certaines initiatives ont permis l'amélioration de l'apprentissage et de l'enseignement. Pour le département de soins infirmiers du cégep de Saint-Félicien, quelques-unes des adaptations mises en place durant la pandémie ont été bénéfiques à l'apprentissage des étudiants. Ils prévoient réutiliser certaines d'entre elles. Par exemple, la plateforme Moodle sera de nouveau exploitée. Elle permet de déposer tous les documents d'un cours à un même endroit, en plus de réduire l'impression de ceux-ci.

La mise en place de boites individuelles pour chaque étudiant contenant le matériel nécessaire à la pratique des soins est aussi une pratique qui perdurera. Elle permet aux étudiants d'être plus autonomes et de conserver les laboratoires propres et rangés. De plus, puisque la pandémie est encore présente, les boites permettent aux étudiants de pratiquer à la maison lorsque leur présence en classe n'est pas permise.

Une autre adaptation appréciée demeure la journée de stage réalisée dans les laboratoires du cégep. Cette journée a été grandement appréciée des étudiants et a permis une meilleure intégration au milieu de stage. Les enseignants pensent utiliser de nouveau cette façon de faire puisque les retombées de cette adaptation sur les étudiants ont été très positives.

Une dernière adaptation qui devrait être de nouveau utilisée se rapporte aux formations en ligne interactives. Les enseignants du département de soins infirmiers pensent les utiliser de nouveau pour la 


\section{$\&$}

\section{REVUE HYBRIDE DE L'ÉDUCATION}

session d'hiver 2021. Selon eux, elles permettent de consolider les notions abordées en classe et peuvent compenser certaines heures de stages.

Pour conclure, la pandémie a bouleversé grandement les pratiques enseignantes. Tous les acteurs du milieu de l'éducation ont dû rapidement s'adapter. L'expérience de l'équipe du département de soins infirmiers du cégep de Saint-Félicien démontre qu'il a fallu beaucoup de créativité, de persévérance et de travail d'équipe pour réussir à offrir une éducation de qualité dans de telles circonstances.

\section{Référence}

Rice, W. et William, H. (2006). Moodle. Packt publishing. 